\title{
Perioperative platelet inhibition in transurethral interventions: TURP/TURB
}

\author{
Michael Wenders, Olaf Wenzel, Thomas Nitzke, Gralf Popken \\ Department of Urology, HELIOS Clinic, Berlin-Buch, Germany
}

\section{ABSTRACT}

Purpose: To determine whether transurethral surgery under platelet inhibition is a feasible procedure. Before transurethral resection of prostate (TURP) or bladder tumours (TURB), the administration of platelet-inhibiting medication is often interrupted due to possible bleeding complications. We studied the performance of TURP and TURB under the current recommendations of the American College of Chest Physicians (ACCP) on perioperative platelet inhibition.

Materials and Methods: Patients assigned for transurethral intervention were preoperatively divided into the following risk groups: low, medium and high cardio- or cerebrovascular risk. In patients with a low-risk profile, acetylsalicylic acid (ASA) was discontinued. Patients of the medium risk group continued taking $100 \mathrm{mg}$ of ASA. Patients of the high-risk group receiving dual platelet inhibition (ASA + clopidogrel) were not treated operatively. In total 346 patients from the low and medium risk groups underwent transurethral intervention.

Results: Forty-two out of 198 TURP were performed under $100 \mathrm{mg}$ of ASA. Without ASA, a significantly shorter length of stay and earlier removal of the transurethral catheter was documented. In the parameters postoperative haemorrhage and operative revision, no significant differences were observed.

Thirty-two out of 148 TURB were performed under $100 \mathrm{mg}$ of ASA. Regarding the length of stay, time until catheter removal, postoperative haemorrhage and operative revision, no significant differences were found under ASA. Only significantly longer continuous irrigation was documented under ASA.

Conclusion: In the case of a verified indication for use of platelet inhibitors, it is possible to avoid discontinuation and the consequent increased risk of thromboembolic incidents in transurethral surgery is admissible.

\section{ARTICLE INFO}

\section{Key words:}

Transurethral Resection of

Prostate; Platelet Aggregation

Inhibitors; Thrombosis

Int Braz J Urol. 2012; 38: 606-10

Submitted for publication:

April 09, 2012

Accepted after revision: July 19, 2012

\section{INTRODUCTION}

In relation to cardiovascular risks, the administration of platelet inhibitors reduces the incidence of perioperative myocardial infarction or stroke by $1 / 3$ (1). Before surgical interventions such as transurethral resection of prostate (TURP) or bladder tumours (TURB), the administration of these medicines is often discontinued due to possible bleeding complications (2). The perioperative discontinuation of platelet inhibitors without checking the indication or their necessity can lead to potentially fatal arterial thromboses (3-5). We studied the performance of TURP and TURB, taking into account the current recommendations of the American College of Chest 
Physicians (ACCP) for perioperative platelet inhibition according to standardized perioperative and postoperative parameters (4).

\section{MATERIALS AND METHODS}

All patients were divided before the transurethral interventions according to the criteria listed below (Table-1) into the following risk groups: low, medium and high cardio- or cerebrovascular risk. The average age of the patients was 68.02 years. In patients with a low-risk profile, administration of the current oral platelet-inhibiting medication (ASA) was discontinued. Patients of the medium-risk group continued to take ASA (clopidogrel was

Table 1 - Perioperative platelet inhibition in transurethral resections.

1) Low cardiovascular or cerebrovascular risk:

- no $\mathrm{CHD} * / \mathrm{PAD}^{*} /$ atherosclerosis

- no cardiovascular stents

- no medium-grade to high-grade vascular stenoses

- no stroke or TIA in medical history

$\Rightarrow$ discontinue ASA $100 \mathrm{mg} 5$ days before surgery until 7 days after the surgery

2) Medium cardiovascular or cerebrovascular risk:

- Prostate volume max. $75 \mathrm{~cm}^{3}$

- CHD / PAD/ vascular stenosis / atherosclerosis

- cardiac stents: DES* > 12 months, BMS $^{*}>6$ weeks

- status post stroke or TIA

- status post myocardial infarction > 12 months

$\Rightarrow$ do not discontinue ASA $100 \mathrm{mg}$

3) High cardiovascular or cerebrovascular risk: ASA and clopidogrel

- status post DES* implantation $<12$ months or BMS* $<6$ weeks

- extracranial and intracranial stent implantation $<6$ weeks

- P3 - synthetic bypass

- status post acute stroke $<12$ months

- critical vascular stenosis

- status post myocardial infarction $<12$ months

=> postponed elective interventions

=> In exceptional cases administration of GPIlb/IIla inhibitors

* DES = drug-eluting stent; $B M S=$ bare metal stent

CHD = Coronary heart disease,

$\mathbf{P A D}=$ Peripheral arterial disease,

TIA = Transient ischaemic attacks 
preoperatively replaced with ASA). Patients of the high-risk group receiving dual platelet inhibition with ASA and clopidogrel were not treated operatively. The following characteristics were studied: days of continuous irrigation, postoperative haemorrhage, length of stay in hospital, days until catheter removal, revision and occurrence of cardio-cerebral event.

Out of 198 prostate resections, 42 were operated under ASA $100 \mathrm{mg}$. During continuation of the platelet-inhibiting medication, a maximum preoperative prostate volume of $75 \mathrm{~mL}$ was determined by transrectal ultrasonography. TURP was performed by low-pressure resection after applying trocar cystostomy under irrigation with Purisole ${ }^{\circledR}$. A 24 Charrière resectoscope was used. The resections were performed following the same protocol until a wide view was obtained. After the completion of the intervention, the 20 Charrière haematuria-irrigation catheter was inserted and blocked in the prostatic fossa at $15 \mathrm{~mL}$. After the surgery, artificial irrigation with $\mathrm{NaCl}$ solution took place for 24 hours which was adjusted by an experienced nursing staff to individual bleeding situations. After that, the irrigation was finished in an optimal case and the catheter was left for a further 24-hour period.

In the case of TURB, 32 patients out of 148 took ASA $100 \mathrm{mg}$. All tumors resected were uni- or multilocular tumours (1-5) of the bladder ranging in size from $3 \mathrm{~mm}$ to $4 \mathrm{~cm}$. There were no differences between the groups. A 24 Charrière resectoscope was used. Except for re-resections and palliative therapy situations, resection was performed by use of photodynamic diagnostics, after instillation of a fluorescent substance. After that, only catheter drainage took place for 24 hours. Irrigation was used only in the case of progressive haematuria.

The results were documented until the day of discharge and subsequently analysed statistically with the Mann-Whitney U-test and Fisher's exact test for significance. Significance was determined at the $p$-value $\leq 0.05$.

\section{RESULTS}

Concerning transurethral resections of the prostate, the average postoperative length of stay of patients who were not on antiplatelet therapy was significantly shorter $(p=0.001)$ : 3.86 days without ASA versus 5.72 days with ASA. Catheter removal could be performed on average after 2.21 days without platelet inhibition and after 2.74 days with platelet inhibition $(p=0.015)$. Postoperative haemorrhage, defined by the presence of clot retention, resumption of bladder irrigation or reinsertion of the urinary catheter, occurred without ASA in 13.5\% of cases, and with ASA significantly more often ( $p=0.032)$ in 34\% of cases. Nine out of 156 patients without ASA had to be submitted for a surgical revision. Under ASA, 5 patients out of 42 had to be reoperated on. In one case haemorrhage requiring transfusion occurred. There was no statistically significant difference in the number of surgical revisions $(\mathrm{p}=0.181)$. The gland volumes were approximately equal, with a mean value of $41.3 \mathrm{~mL}$ vs. $39.6 \mathrm{~mL}$. No cardiovascular events occurred in the total patient population.

In the case of transurethral resections of the urinary bladder, no significant difference ( $p$ $=0.238$ ) was found in the average postoperative length of stay of patients who were not under platelet inhibition (2.91 days versus 3.34 days under ASA). Catheter removal could be performed on average after 1.27 days without platelet inhibition, and after 1.5 days with platelet inhibition ( $p$ $=0.47$ ). Postoperative haemorrhage occurred without ASA in $8.6 \%$ and with ASA in $18.7 \%$ of cases. The difference was not significant $(p=0.115)$.

Four out of 116 patients without platelet inhibition had to be submitted to a surgical revision. In the ASA group, 2 patients out of 32 had to be reoperated on. Again there was no significant difference $(p=0.61)$.

However, under ASA there was significantly longer continuous irrigation: 0.625 days versus 0.267 days without platelet-inhibiting medication. No cardiovascular events occurred in the total patient population.

\section{DISCUSSION}

Patients who are submitted to a transurethral intervention are often in an advanced age with corresponding cardiovascular risk factors or diseases. For example, Wasson et al. reported that 
in a series of 280 urological patients in the USA with an average age of 66 , in 42 patients a coronary heart disease was present (6). These patients often receive antiplatelet medication with ASA and/or clopidogrel $(3,7)$. The administration of such platelet inhibitors reduces by one third the incidence of myocardial infarct as well as stroke in patients with cardiovascular risks or diseases (1). The unverified discontinuation of this medication may lead, however, to life-threatening iatrogenic coagulopathies such as stent thromboses (3-5). Even though the problems of perioperative discontinuation of such medication have frequently been described in the guidelines, there is no guideline for high-risk cardiovascular patients on antiplatelet medication before non-cardiac surgical interventions.

Since intraoperative bleeding during transurethral resection of the prostate or urinary bladder complicates the technical execution of these interventions and postoperative bleeding potentially leads to the necessity of blood transfusions, revisions and longer stay in hospital, the administration of such medication is often discontinued or avoided for fear of bleeding complications. Enver et al. reported in this context that 178 out of 287 urologists in the UK asked their patients to stop taking platelet inhibitors before prostate resection (TURP). Among these 178 urologists, 62\% terminated the medication without verifying the indication for these medicines (2).

In the currently available literature, increased bleeding tendencies and blood transfusion rates under aspirin are described in two studies referring to TURP $(8,9)$. A prospective, randomized and placebo-controlled study by Nielsen JD et al. revealed no significant intraoperative blood loss under $150 \mathrm{mg}$ of ASA, but described significantly increased postoperative blood loss under ASA (10). Based on these studies, discontinuation of ASA before the surgical intervention was recommended. Ala-Opas et al. found by comparison of 40 patients under ASA versus 42 patients without oral platelet inhibition no difference in the average blood loss and thus saw no contradictions for TURP under ASA (11).

Our results support the reports of an increased bleeding tendency in transurethral interventions under ASA. However, they also demonstrate that under ASA serious bleeding necessitating revision does not occur significantly more often. In summary, discontinuation and thereby an increased risk of thromboembolic incidents can be avoided if there is a verified indication for a platelet-inhibiting medication.

The investigated groups differ regarding their risk categories. This might have had an impact on long term outcome since the comorbidities of patients taking platelet inhibitors are usually higher. Since our study focused on very specific short term peri- and postoperative results the authors did not expect any relevant bias. However, long term studies with a prospective randomized and double blinded study design should be initiated.

\section{CONCLUSIONS}

Based on the results, we can conclude that the performance of transurethral resection under oral platelet inhibition with ASA $100 \mathrm{mg}$, under strict observance of the criteria listed in Table-1, is justified and therefore viable.

\section{CONFLICT OF INTEREST}

None declared.

\section{REFERENCES}

1. [No authors listed]: Collaborative overview of randomised trials of antiplatelet therapy--l: Prevention of death, myocardial infarction, and stroke by prolonged antiplatelet therapy in various categories of patients. Antiplatelet Trialists' Collaboration. BMJ. 1994; 308: 81-106. Erratum in: BMJ 1994; 308: 1540.

2. Enver MK, Hoh I, Chinegwundoh Fl: The management of aspirin in transurethral prostatectomy: current practice in the UK. Ann $\mathrm{R}$ Coll Surg Engl. 2006; 88: 280-3.

3. Shahar E, Folsom AR, Romm FJ, Bisgard KM, Metcalf PA, Crum $L$, et al.: Patterns of aspirin use in middle-aged adults: the Atherosclerosis Risk in Communities (ARIC) Study. Am Heart J. 1996; 131: 915-22.

4. Grines CL, Bonow RO, Casey DE Jr, Gardner TJ, Lockhart PB Moliterno DJ: Prevention of premature discontinuation of dual antiplatelet therapy in patients with coronary artery stents: a science advisory from the American Heart Association, American College of Cardiology, Society for Cardiovascular Angiography and Interventions, American College of Surgeons, and American Dental Association, with representation from the American College of Physicians. Circulation. 2007; 115: 813-8. 
5. [No authors listed]: Antithrombotic Trialists' Collaboration Collaborative meta-analysis of randomised trials of antiplatelet therapy for prevention of death, myocardial infarction, and stroke in high risk patients. BMJ. 2002; 324: 71-86. Erratum in: BMJ. 2002; 324: 141.

6. Wasson JH, Reda DJ, Bruskewitz RC, Elinson J, Keller AM, Henderson WG: A comparison of transurethral surgery with watchful waiting for moderate symptoms of benign prostatic hyperplasia. The Veterans Affairs Cooperative Study Group on Transurethral Resection of the Prostate. N Engl J Med. 1995; 332: 75-9.

7. Vasudeva P, Goel A, Sengottayan VK, Sankhwar S, Dalela D: Antiplatelet drugs and the perioperative period: What every urologist needs to know. Indian J Urol. 2009; 25: 296-301.

8. Wierød FS, Frandsen NJ, Jacobsen JD, Hartvigsen A, Olsen PR: Risk of haemorrhage from transurethral prostatectomy in acetylsalicylic acid and NSAID-treated patients. Scand J Urol Nephrol. 1998; 32: 120-2.
9. Thurston AV, Briant SL: Aspirin and post-prostatectomy haemorrhage. Br J Urol. 1993; 71: 574-6.

10. Nielsen JD, Holm-Nielsen A, Jespersen J, Vinther CC, Settgast IW, Gram J: The effect of low-dose acetylsalicylic acid on bleeding after transurethral prostatectomy--a prospective, randomized, double-blind, placebo-controlled study. Scand J Urol Nephrol. 2000; 34: 194-8.

11. Ala-Opas MY, Grönlund SS: Blood loss in long-term aspirin users undergoing transurethral prostatectomy. Scand J Urol Nephrol. 1996; 30: 203-6.

Correspondence address: Dr. Michael Wenders Klinik für Urologie HELIOS Klinikum Berlin-Buch, Schwanebecker Chaussee 50, 13125 Berlin, Germany.

Fax: +49 30 9401-5250

E-mail: m.wenders@web.de 